\begin{abstract}
This study examines how social cues facilitate learning by manipulating the familiarity of a social cue. Participants were forty-nine infants between 12-18 months. Infants were taught a novel label for a novel object under two pre-recorded gaze conditions - one in which the caregiver was seen gazing at a novel object while a verbal label was played, and one in which a stranger was seen gazing at a novel object while a verbal label was played. Learning was only evident in the caregiver condition and only in the infants most skilled at following their caregivers' gaze. The results of the current study suggest that both the familiarity of the cuer and the infant's own ability to follow the gaze of the cuer play important roles in the infant's learning in this task.
\end{abstract}

Keywords: gaze following, social cues, attention, eye-tracking, infancy 
Caregivers and Strangers: The Influence of Familiarity on Gaze Following and Learning

Even the youngest infants possess a simple heuristic that helps them attend to highly relevant information in the social environment. Newborn infants prefer to look at face-like stimuli over stimuli not organized like a face (Goren, Sarty, \& Wu, 1975; Johnson, Dziurawiec, Ellis, \& Morton, 1991). Interestingly, the early preference for faces is coupled with an early preference for familiarity. Within the first days, infants show a visual preference for the mother's face over a stranger's face (Bushnell, Sai, \& Mullin, 1989; Bushnell, 2001; Field, Cohen, Garcia, \& Greenberg, 1984; Pascalis, de Schonen, Morton, Deruelle, \& Fabre-Grenet, 1995). The behavioral preference for the mother's face is not restricted to neonates. Infants prefer this highly familiar face over an unfamiliar face throughout the first year (Barrera \& Maurer, 1981) (Wagner, Luyster, Yim, Tager-Flusberg, \& Nelson, 2013).

An important characteristic of faces is that they provide infants with a great deal of information about their environments. When navigating complex environments, infants can use the direction of adults' attention to direct their own attention to what is important and relevant in the moment. Rudimentary gaze following can be seen in infants as early as two days after birth (Farroni, Massaccesi, \& Johnson, 2004). Newborns can locate a peripheral target cued by a shift in pupil position on a schematic face. More complex gaze following emerges by three months. At this age, infants can follow an experimenter's head turn toward stationary toys located in close proximity to the infant (D’Entremont, 2000; D’Entremont, Hains, \& Muir, 1997). By the end of the first year, infants can localize the specific target of an adult's gaze in the presence of multiple potential targets (Butterworth \& Jarrett, 1991). This has also been seen when following an adult's gaze on a screen (Von Hofsten, Dahlstrom, \& Fredriksson, 2005), suggesting that an infant does not need to interact with the attention-director directly in order to follow her gaze. By 
18 months, infants' localization abilities improve to the point that they can even follow an adult's gaze to a target located out of their own line of sight (Butterworth \& Jarrett, 1991).

When an infant follows an adult's gaze toward an object or event in the environment, it presents an opportunity to then learn. By twelve months, infants can use the direction of an adult's gaze to associate a label with the correct object (Hollich et al., 2000). Their abilities become more sophisticated over the second year, and by 24 months, infants can map a label onto an object indicated by an adult's gaze cue even when perceptual salience cues (such as bright color or object movement) draw attention to a different object (Hollich et al., 2000; Moore, Angelopoulos, \& Bennett, 1999). Furthermore, there is evidence that early joint attention ability predicts later language ability (Brooks \& Meltzoff, 2005; Carpenter, Nagell, Tomasello, Butterworth, \& Moore, 1998; Morales et al., 2000), raising the possibility that better use of social cues allows for more learning in an inherently social world. Experimental evidence of this is rare and primarily exists in the form of demonstrations that infants learn best when social cues (including gaze cues) are available rather than unavailable (e.g., Baldwin et al., 1996; Bannard \& Tomasello, 2012). Hirotani, Stets, Striano, \& Friederici (2009) even found that learning of novel label/object associations was enhanced in 18-21 month olds when the words were taught in a joint attention context (defined as the experimenter establishing eye contact and using positive facial and vocal expressions) compared to a non-joint attention context (defined as the experimenter looking to the infant's chest and using neutral facial and vocal expressions). What is not yet clear is how an infant's ability to use gaze cues relates to how well they learn from those cues. Examining the relationship between attention to and learning from a cue, such as the direction of another's gaze, can allow for a better understanding of how attention and learning work together in an infant's daily life. 
It is clear from research with very young infants that a preference for familiar faces (mothers) emerges early in life and persists throughout the first year of life (Barrera \& Maurer, 1981; Wagner et al., 2013), but we do not yet know how familiarity with a face affects the ability to use gaze cues during learning. Because of the sheer volume of time infants spend with their caregivers, it is likely that they see these familiar faces performing common actions (e.g., show a fearful expression or look towards objects) more frequently than they see unfamiliar faces performing the same actions. The preference for familiar faces reported during the first year, therefore, may extend to actions that those faces perform. Furthermore, infants may process information provided by familiar faces differently than information provided by unfamiliar faces. Little research has examined how infants differentially use information they garner from familiar and unfamiliar agents in their environments. Investigating processing of information from familiar and unfamiliar agents is necessary to elucidate the impact that familiarity has on infants' learning about the surrounding environment.

Hoehl, Wahl, Michel, and Striano (2012) conducted a study that investigated how young infants differentially use information provided by familiar and unfamiliar agents in their environments. Four-month-old infants viewed photographs of their own caregiver and a stranger looking toward or away from a novel object. They were then shown the object on the screen alone. Electrophysiological evidence (ERP responses) suggest that objects the parent gazed away from required additional encoding (indexed by a greater positive slow wave response) than objects gaze-cued by the parent. There were no differences in the positive slow wave for the objects from which the stranger looked toward or away. The results suggest that the familiarity of the parent's face allowed for more thorough processing on trials during which the parent's gaze cued the object. Hoehl and colleagues (2012) suggested that the extensive experience 
infants have with their caregivers, in comparison to unfamiliar adults, allows the infants to more quickly encode the caregiver's face and then use that face to guide attention and process the objects. It was suggested that the more rapid identification and encoding of the face had the potential to facilitate the processes that happen subsequent to face encoding.

Only one study has directly compared how the identity of the person directing attention affects gaze following. Gredebäck, Fikee, and Melinder (2010) tested infants at two, four, six, and eight months. In the task, infants watched either their own mother or a stranger gaze toward one of two toys on a tabletop in front of the child. At six months, infants who watched a stranger exhibited more gaze following than infants who watched their own mothers. The authors suggest that the infants were more interested in the stranger, and this led them to follow gaze more in this condition. The results from Gredebäck et al. suggest young infants' preference for following the gaze of unfamiliar adults, which conflicts with the explanation provided by Hoehl et al. (2012). Hoehl et al.'s (2012) findings suggest that following the caregiver's gaze may be better for learning about objects, while paradoxically, the results of Gredebäck et al.'s experiment (2010) suggest that infants prefer to follow the gaze of a stranger. It is not clear why the experiments present conflicting findings, however it is important to consider that the two experiments used different tasks and methods. Hoehl et al.'s experiment was conducted on a screen and responses were measured via ERP. Gredebäck et al.'s experiment, was conducted live and responses were measured via eye tracking. This discrepancy in the literature represents a barrier to understanding the role that familiarity has on infant learning. To address this paradox in the literature, the current study examines both how infants use gaze cues from familiar and unfamiliar people and, within the same experiment, how those patterns of responding to gaze differentially affect learning. 


\section{The Current Experiment}

In the present study, we examined the relationship between word learning and gaze following from a familiar and an unfamiliar person. We used a word learning task because there are several longitudinal studies that have shown a relationship between early gaze following abilities and vocabulary comprehension or production (Brooks \& Meltzoff, 2005; Carpenter et al., 1998; Morales et al., 2000). Infants 12 to 18 months of age were presented with a novel label for a novel object from both the direction of a familiar agent's gaze (i.e., the caregiver) and that of an unfamiliar adult. The infants tested in the current study were older than those previously studied by either Gredebäck et al. (2010) or Hoehl et al. (2012) because the task is more difficult than simply processing the surface characteristics of a novel object (like the task presented by Hoehl et al., 2012). However, the labeling task is ideal for examining the relationship between gaze following and word learning. Further, in the second year of life, infants have ample experience with adults directing their attention to the most relevant information in the environment, and most of this experience is likely to come from their caregivers.

In the current study, we asked three questions: (1) Does infants' familiarity with the caregiver foster better gaze following compared to responding to strangers' gaze cues? (2) Does infants' extensive experience with caregivers promote more efficient learning compared to learning from strangers? (3) How does familiarity interact with an infant's gaze following ability to predict word-learning within a task?

We predicted that infants would more reliably follow the gaze of a caregiver than the gaze of a stranger. Infants have rich prior experience with caregivers' gaze as a useful source of information and are likely to interpret it rapidly. Alternatively, infants at 12 to 18 months of age 
may be so efficient at gaze following that the identity of the gaze cuer does not affect infants' gaze following.

We also predicted that infants would be more likely to learn a label when using the gaze direction of their caregiver to direct their attention than when using the gaze direction of a stranger. If infants more reliably use their caregivers' gaze to guide attention than the gaze of a stranger, then they should also process the information to which they are being directed more efficiently. The broad experience infants have with their caregivers directing attention should make it easier for infants to interpret and use the caregivers' gaze cues for learning in comparison to a stranger's gaze cues.

\section{Method}

\section{Participants}

Forty-nine full-term monolingual infants ( 28 female) between the ages of 12 to 18 months (mean age $=15$ months 26 days; age range $=12$ months 11 days to 18 months 29 days) comprised the final sample. Thirty-eight of the infants were Caucasian, 1 was Asian, and 10 were more than one race. All of the infants' mothers had graduated from high school. Seventy-three percent of the mothers had earned at least a bachelor's degree. An additional 22 infants were tested but excluded from the final sample due to fussiness (8), failure to complete the minimum requirement of 2 valid test trials per condition (4), or because the parent recordings did not meet criteria (10; described below). The mean age of the infants excluded from the final sample did not differ significantly from the included infants (15 months 22 days, $t(40)=0.29, p=0.77, d=$

0.08). Infants were recruited through letters to families in the surrounding area and given a book for participating. The Institutional Review Board approved the experimental protocol, and informed consent was obtained from a parent of each infant. 


\section{Apparatus}

Stimuli were presented on a 17-inch Tobii 1750 LCD binocular eye tracker (1280 x 1024 pixels resolution) to record infants' fixations during the task. Eye tracking data were collected at a sample rate of $50 \mathrm{~Hz}$. The average accuracy of the recorded eye coordinates was about $0.5^{\circ}$, which is approximately $0.5 \mathrm{~cm}$ at a viewing distance of $60 \mathrm{~cm}$. The average accuracy in timing was 25-35 ms. Drifts are compensated with an average error of $0.5^{\circ}$. When one eye could not be measured, data from the other eye were used to determine the gaze coordinates. The recovery time to full tracking ability after an offset was about $100 \mathrm{~ms}$. Stimuli were displayed using the EPrime software (Psychology Software Tools, Pittsburgh, PA), and sounds were presented through external speakers.

\section{Procedure}

We used a within-subjects design. Infants were presented with two novel object labels. Infants were taught a novel label for a novel object under two pre-recorded gaze conditions - one in which the caregiver was seen gazing at a novel object while a verbal label was played, and one in which a stranger was seen gazing at a novel object while a verbal label was played. Importantly, the stranger used for each participant was another child's caregiver that had previously participated in the study. Therefore, the term "stranger" is relative to the particular infant watching the video. Recorded participants were matched only on the presence of glasses and the gender of the caregiver present (43 mothers and 6 fathers). Nearly all caregivers served as a stranger for another baby. However, some parents did not consent to use their video for another participant. 


\section{Stimuli}

Caregiver/director videos. Prior to eyetracking, the infant's caregiver was seated in front of a black background and a black cloth was placed over his/her shoulders to conceal his/her clothing. If the parent had long hair, he/she was instructed to push it behind his/her shoulders. The parent's hands were positioned in a fixed location, outside the camera's range, to the immediate left and right side of his/her body with elbows tucked in at the waist. The recording began with the caregiver looking forward and smiling into the camera, which was approximately three feet in front of him/her. The parent was then instructed to smile, turn and

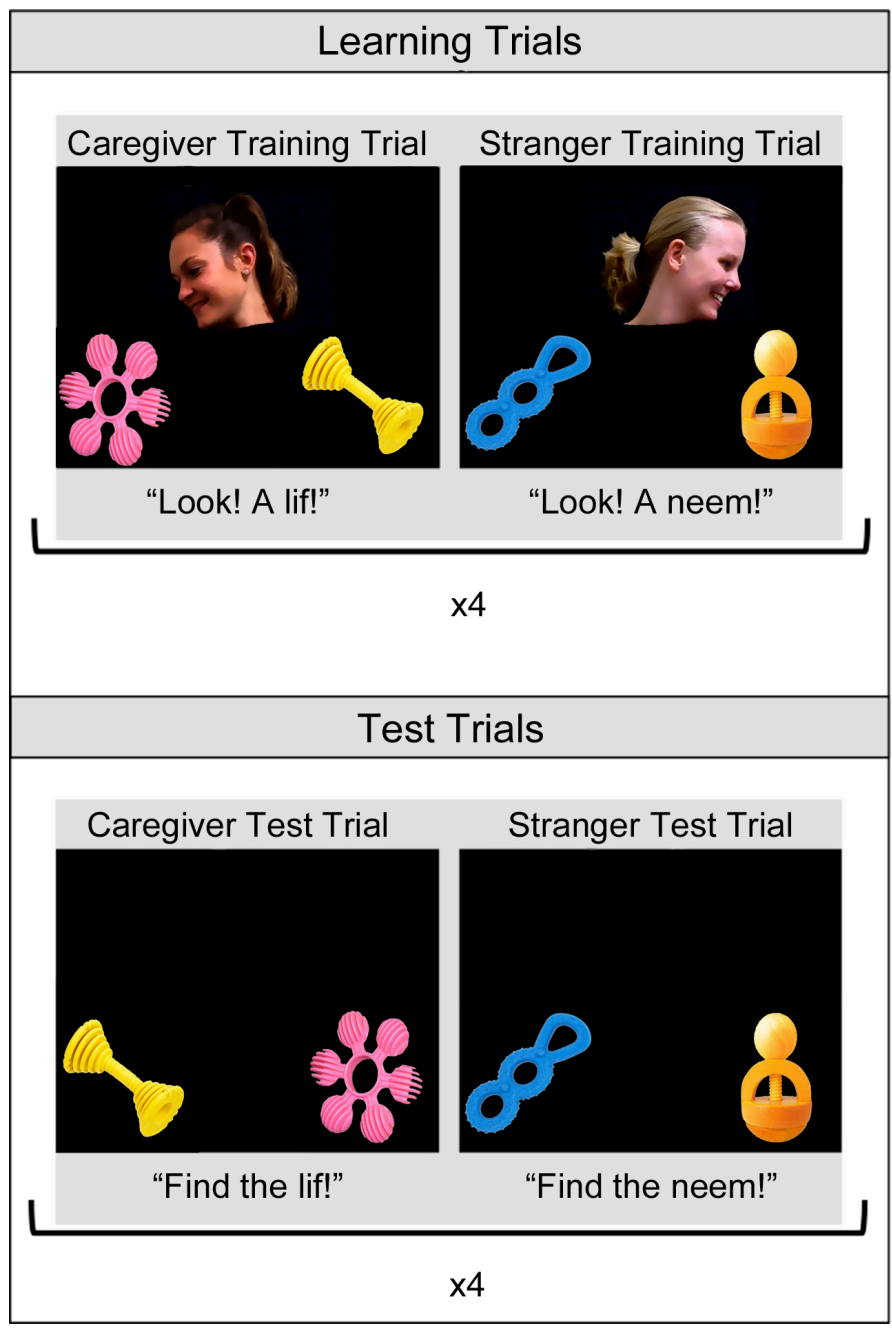

Figure 1. Example stimuli. 
gaze toward his/her left hand, remain there for four seconds, and then turn back to the center and look into the camera. This procedure was repeated with a right head turn. Multiple videos were taken when necessary in order to ensure an accurate video of the caregiver's head turning to view something on the left and right side of his/her body (see Figure 1).

Eyetracking videos. For all eyetracking trials, infants saw a pair of two novel objects, one object in each bottom corner of the screen, as shown in Figure 1. There were four possible objects used. Each object was a minimum of $6 \times 10.5 \mathrm{~cm}$ (subtending $5.73 \times 10^{\circ}$ visual angle). Any of the objects could be paired with any of the other objects, and all infants saw all four objects. Object pairs were assigned to infants randomly. Pairs were counterbalanced between infants. Within infants, however, objects always remained in their assigned pairs. All stimuli were against a black background.

Training Trials. For the training trials, infants saw two of the novel objects on the screen alone for $1500 \mathrm{~ms}$, accompanied by the word "Look!" played through the speakers. Then a centrally located video appeared of a person (the director; i.e., their caregiver or a stranger) who smiled, looked straight ahead, and then turned her head toward one of two novel objects (the target) at the bottom corners of the screen. She remained looking at the target until the labeling was complete. She then turned her head back to the center and the trial ended. The director videos were approximately $8 \times 11 \mathrm{~cm}$, and they subtended approximately $7.63 \times 10.48^{\circ}$ visual angle. The objects remained on the screen throughout the duration of the trial $(8500 \mathrm{~ms})$, and the video of the director was present for $7000 \mathrm{~ms}$. Importantly, only the target object of each pair was ever looked at and labeled.

While the initial head turn was being completed, an audio track played from the speakers. The infant heard the label twice during each trial (e.g., "Look! A lif! It's a lif!"). Each labeling 
passage lasted approximately 2500ms. There were two labels ("lif" and "neem"), and they were randomized between participants so that for half of the infants, the caregiver was paired with the label "lif" and the stranger with the label "neem," and vice versa for the other half. Therefore, the caregiver and stranger did not produce the labels. They simply directed attention to the object while a novel label was presented. The label presentations were kept consistent across caregiver/stranger conditions to allow us to determine if it was the gaze cues of the caregiver and stranger (and not the variations in labeling) that were responsible for the predicted differences in attention and learning. The same audio track was played for all infants. A female with a local American accent recorded the track using infant-directed speech.

There were eight training trials: four caregiver trials and four stranger trials. The order of caregiver and stranger trials was randomized with the constraint that the same type of trial could not appear more than twice sequentially. The target side was also randomized within participants such that each object appeared equally on the left and right.

Test Trials. Following the training trials, infants watched eight test trials: four test trials showing the objects trained by the caregiver and four test trials showing the objects trained by the stranger. Again, the order of trials was randomized with the constraint that the same type of trial could not appear more than twice sequentially. The target side was again randomized within participants. The infant heard a prompt (e.g., "Find the lif/neem") while only the two objects were on the screen without a director present. Each test trial lasted $6000 \mathrm{~ms}$. The objects appeared at the start of the trial along with a single "Look!" followed by $2000 \mathrm{~ms}$ of silence and then the prompt to find the object. 
Eight training trials followed by eight test trials made up a single block (see Figure 1). Two blocks were presented. However, an infant did not need to complete two blocks to be included in the analysis.

\section{Data Preparation}

Training Phase. Gaze Replay movies were exported from Tobii Studio. These movies include the location of the infant's gaze pattern overlaid onto the video the infant watched. The movies were coded frame-by-frame using Datavyu software (Datavyu Team, 2014). Only videos in which the director (caregiver or stranger) looked directly ahead for at least $300 \mathrm{~ms}$ before gaze cueing were included in the final analysis because eye contact has been found to enhance gaze following (de Bordes, Cox, Hasselman, \& Cillessen, 2013; Senju \& Csibra, 2008). A value of $300 \mathrm{~ms}$ was chosen because this is the minimum length of time an infant would need to be able to plan a saccade to the director's face after noticing the appearance of a face (Haith, Wentworth, $\&$ Canfield, 1993). Further, only trials during which the infant fixated on the director's face during the head turn were included in the final analysis because the head turn motion enhances gaze following (Deak, Flom, \& Pick, 2000; Moore, Angelopoulos, \& Bennett, 1997).

The first author coded all of the videos. The coder was blind to the identity of the directors while coding. Two types of events were coded: director's behavior (onset and offsets of head turns) and infant's eye gaze (looks to the target object, looks to the distractor object, and looks to the director). The onset of a head turn was defined as the point at which the head began to rotate in the direction of the target object. The offset of a head turn was defined as the point at which the head began to rotate back toward the center. This onset and offset time allowed for the trial to be divided into three distinct phases: pre-cueing (the time before the head turn was initiated), cueing (the time from when the head turn was initiated until the director began 
returning to the center), and post-cueing (the time after the director began returning to the center).

For infants' looks to the target and distractor objects, we coded the onset and offset time of the infants' gaze, the previous gaze location (target object, distractor object, director, or unknown), and the phase in which the look occurred (pre-cueing, cueing, or post-cueing). For looks to the director, we coded the onset and offset time of the infants' gaze and the previous gaze location (target object, distractor object, or unknown). The phase was not included for looks to the director because infants often continued to look to the director during phase transitions.

A gaze shift was defined as a shift from the director's face to an object during the cueing phase (as in Gredebäck et al., 2010). The proportion of accurate gaze shifts (including all gaze shifts within each trial) was calculated as the number of accurate shifts (shifts to the target object) divided by the total number of accurate and inaccurate shifts (shifts to the distractor object). Data were averaged across trials to create a single accuracy score for each condition for each infant.

Latency of the first face to object gaze shift is also reported. Latency was calculated by subtracting the time when the infant first fixates on the director's face during the cueing phase from the time when the infant first fixates on the target object (following the initial fixation on the director's face). Data were averaged across trials to create a single latency value for each condition for each infant.

Reliability Coding. A second coder (who was blind to study hypotheses and the identity of the directors) coded $25 \%$ of the videos. Inter-observer reliability was assessed by calculating the mean proportion of shifts in gaze (from either the target object, distractor object, or director) in which coders agreed within one frame (33ms). Coders agreed within one frame on $89 \%$ of all 
shifts. When disagreements arose, the first coder re-examined those segments and re-coded as necessary.

Test Phase. We only included trials in which the infant was fixating on an object at the label onset, consistent with studies using similar test trials (e.g., Fernald, Perfors, \& Marchman, 2006; Fernald, Pinto, Swingley, Weinberg, \& McRoberts, 1998). Infants provided an average of $5.71(S E=0.26)$ valid trials in the caregiver condition and an average of $5.33(S E=0.27)$ valid trials in the stranger condition. A paired samples $t$-test revealed that these were not significantly different, $t(48)=1.76, p=0.09, d=0.21$. Accuracy of looking was calculated by dividing the time spent looking at the target object divided by the time spent looking at both the target and distractor objects over the window of 300-1800ms after the onset of the target label. The analysis window began $300 \mathrm{~ms}$ after the onset of the label because shifts occurring before this time likely originated prior to the onset of the label (Haith et al., 1993). An analysis window of 300-1800ms has been used in prior studies using similar age groups (e.g., Fernald et al., 2006; Fernald, Zangl, Portillo, \& Marchman, 2008). Data were averaged across trials for each infant to create a single accuracy score for each condition in each block.

\section{Results}

Because we did not have specific hypotheses regarding infant age, this variable was used as a covariate in each omnibus analysis of covariance (ANCOVA). It was necessary to meancenter age, as this prevents the between-subjects covariate from altering within-subjects main effects (Delaney \& Maxwell, 1981). We also did not have specific hypotheses regarding the gender of the caregiver/stranger pair, but because the number of females and males serving as cues was unequal, this variable was included in each omnibus ANCOVA. 


\section{Training Phase}

The effect of familiarity on infants' gaze following behavior was examined by analyzing looking time, gaze shift accuracy, and latency to fixate on the target object during the training phase. The analytical design included condition (caregiver or stranger) as a within-subjects factor, gender of the caregiver and stranger pair (male or female) as a between-subjects factor, and mean-centered age as a covariate.

Looking time. We used a repeated measures ANCOVA to analyze looking time to the director, target object, and distractor object during the cueing phase to determine if the identity of the director affects how infants allocate their visual attention during the training phase, while controlling for age. Average looking time (in milliseconds) was entered into the ANCOVA with one additional within-subjects factor: Area of Interest (AOI; director, target, or distractor object). The degrees of freedom were corrected using the Huynh-Feldt correction because the assumption of sphericity was violated. The analysis revealed a significant main effect of AOI, $F(1.10,50.77)$ $=263.57, p<0.001, \eta_{p}^{2}=0.85$. Post hoc comparisons applying Bonferonni correction revealed that infants looked to the director $(M=3238.14 \mathrm{~ms}, S E=101.56)$ significantly longer than they looked to the target object $(M=222.66 \mathrm{~ms}, S E=25.61, p<.001)$ or the distractor object $(M=$ $197.76 \mathrm{~ms}, S E=25.23, p<.001)$. However, infants looked to the target and distractor objects for a similar length of time $(p=0.85$, see Figure 2$)$. There was a significant effect of age, $F(1,46)=$ $5.29, p=0.03, \eta_{p}^{2}=0.10$, such that the average time spent looking to the screen increased with age. There was also a significant AOI by age interaction, $F(1.10,50.77)=4.87, p=0.03, \eta_{p}^{2}=$ 0.10 , such that time spent looking at the face increased with age, $r(49)=0.31, p=0.03$. Age was not significantly correlated with time spent looking at the target object, $r(49)=-0.29, p=0.05$, or distractor object, $r(49)=-0.10, p=0.48$. There was no significant main effect of condition or 
gender of the caregiver/stranger pair, no significant interactions of condition by AOI, AOI by gender of caregiver/stranger pair, condition by gender of caregiver/stranger pair, or AOI by condition by gender of caregiver/stranger pair, all $F$ 's $<1$.

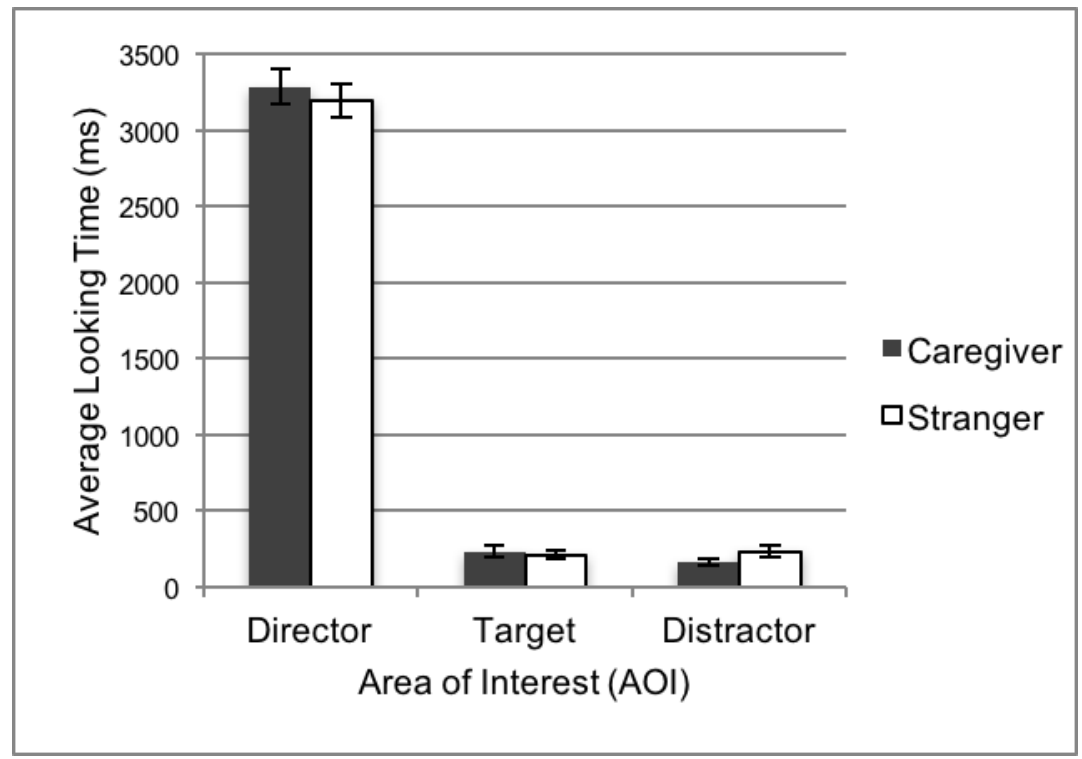

Figure 2. Average looking time to each AOI during the training phase for each condition. Error bars represent Standard Errors.

Gaze shifts. By coding individual gaze shifts as accurate (i.e., to the cued target) or inaccurate, we can better characterize the manner in which the infants used the cues to direct their attention. Each infant saw at least 8 trials, and infants had approximately five seconds to shift gaze within a trial. There were 8 infants who showed no gaze following in one or both conditions (5 in the caregiver condition only; 1 in the stranger condition only; 2 in both conditions).

If an infant never shifted her gaze from the director to one of the objects, that infant's data were not removed from the current analysis. Instead, that infant received an accuracy score of 0 . Seven infants never shifted their gaze from their caregivers' face to an object. Only three infants never shifted their gaze from the stranger's face to an object, and two of these infants were a subset of the seven who never shifted from their caregivers' face. Therefore, a total of 
eight infants received a score of 0 for never shifting from one or both directors. Accuracy of gaze shifts was used as the dependent variable in the repeated measures ANCOVA. The analysis revealed no significant effect of condition, gender of the caregiver/stranger pair, or age, all $F$ 's $<1$.

Next, we compared the accuracy of gaze shifts against chance (where a chance score would be 0.50 indicating equal number of shifts to the target and distractor objects) for both conditions separately to determine if infants were reliably following the cuers gaze to the target object (see Figure 3 for the distribution of accuracy scores in each condition). Infants did not show above-chance gaze following in the caregiver condition $(M=0.53, S E=0.05 ; t(48)=0.58$, $p=0.57, d=0.08)$ or the stranger condition $(M=0.57, S E=0.05 ; t(48)=1.51, p=0.14, d=$ 0.21). It should be noted, however, that if you remove the eight infants who did not shift from their caregiver or the stranger at all, the remaining infants showed reliable shifting responses to their caregivers $(M$ $=0.61, S E=0.05 ; t(40)=2.36$, $p=0.02, d=0.37)$ and strangers $(M=0.62, S E=0.05 ; t(40)=$ $2.45, p=0.02, d=0.38)$. The result of the repeated measures ANCOVA, however, remained

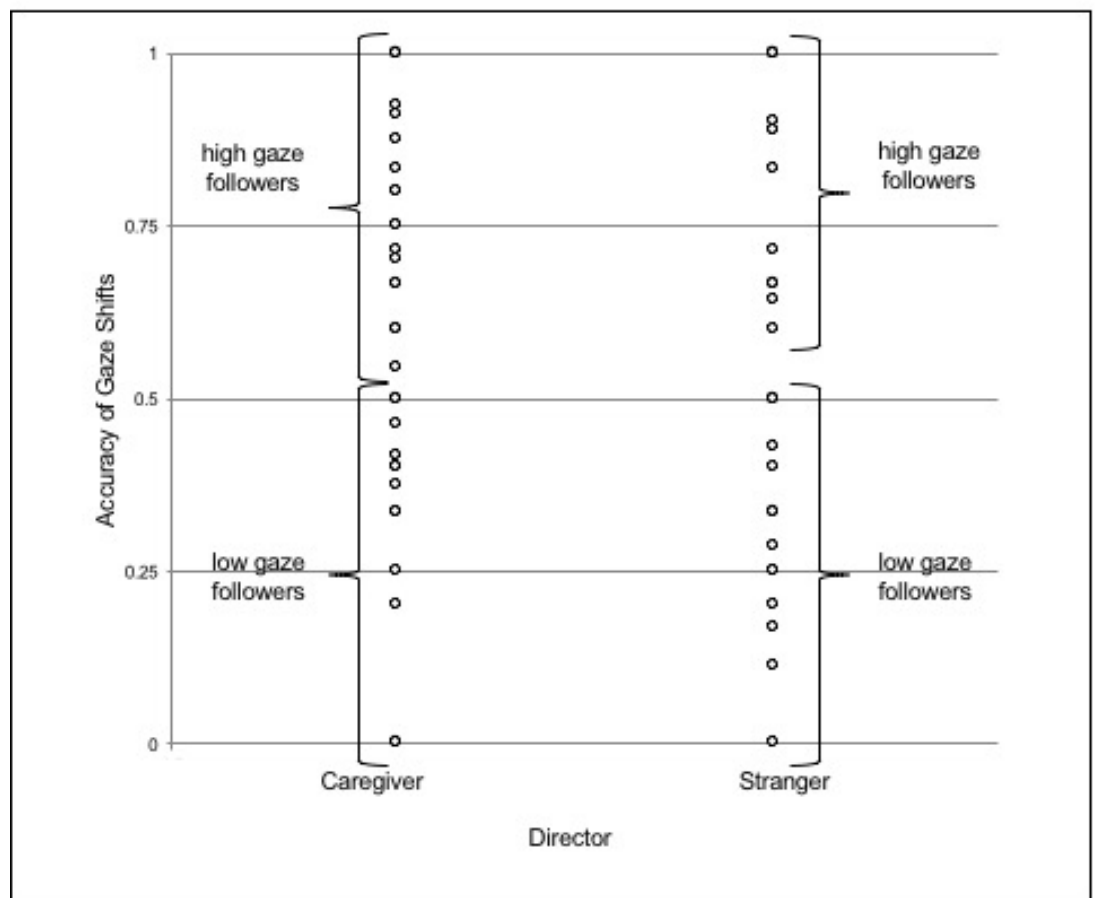

Figure 3. Average gaze shift accuracy score during the training phase for all infants. The proportion of accurate gaze shifts (including all gaze shifts within each trial) was calculated as the number of accurate shifts (shifts to the target object) divided by the total number of accurate and inaccurate shifts (shifts to the distractor object). 
the same (all F's <1) with those infants removed.

Finally, we examined the correlations between infants' gaze shift accuracy scores from the caregiver and those from the stranger to determine if gaze following ability was a characteristic of individual infants, regardless of familiarity of the director. The accuracy scores were not correlated, $r(47)=.08, p=.58($ see Figure 4$)$.

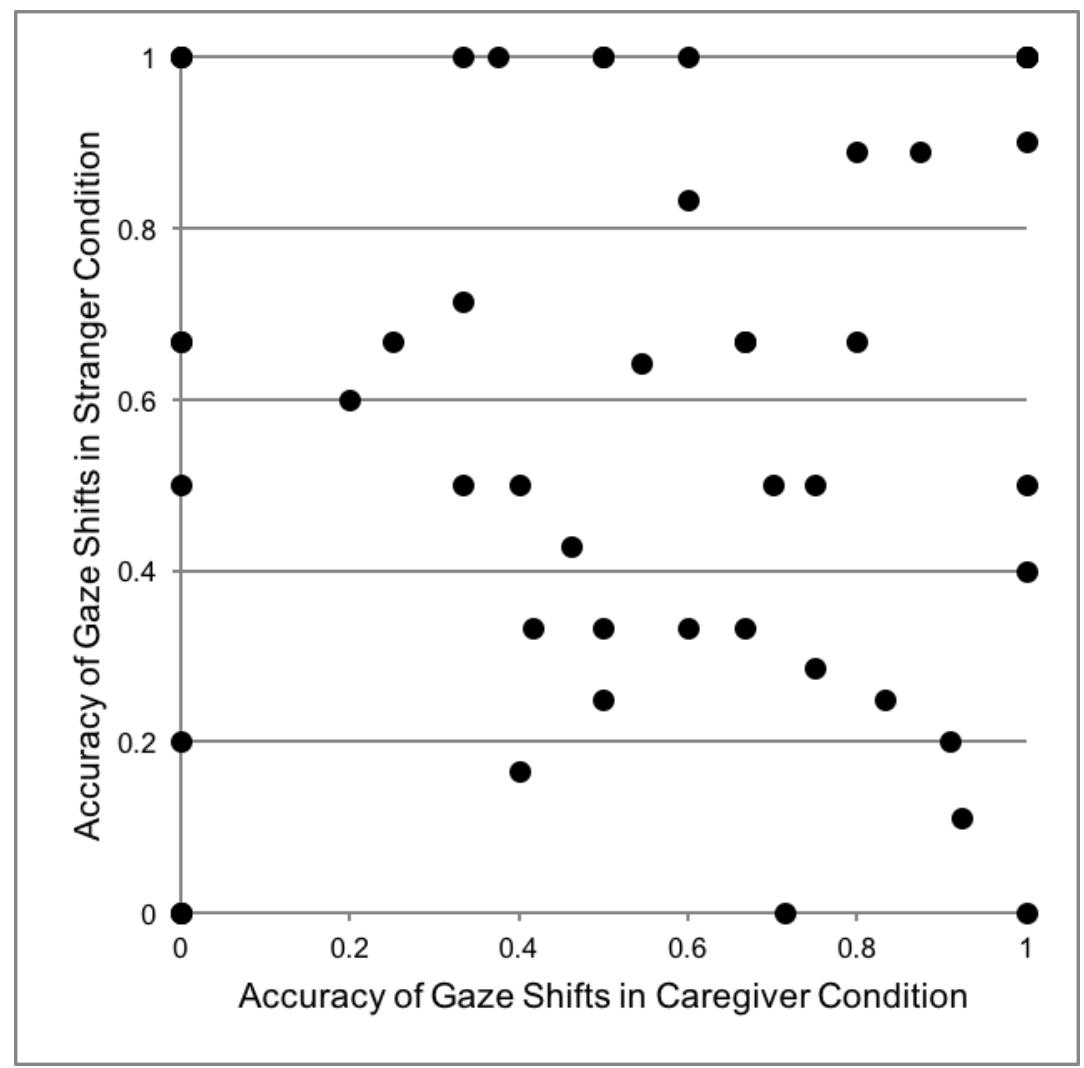

Figure 4. Scatterplot of average gaze shift accuracy scores during the training phase in each condition

Latency. To examine the effect of familiarity on how quickly infants shift attention from the director to the target object, we entered latency to fixate on the target object into a repeatedmeasures ANCOVA. The analysis revealed no significant main effect of condition when controlling for infant age, $F<1$. The latency during caregiver trials $(M=2652.03 \mathrm{~ms}, S E=$ 154.10) was similar to the latency for stranger trials $(M=2425.62 \mathrm{~ms}, S E=169.94)$. There was 
also no significant effect of age, $F(1,38)=4.16, p=0.05, \eta_{p}^{2}=0.10$, gender of caregiver/stranger pair, $F(1,38)=4.19, p=0.05, \eta_{p}^{2}=0.10$, or condition by gender of caregiver/stranger pair interaction, $F<1$.

Summary. No significant condition effects were evident across the analyses.

Infants' looking times were similar in both conditions. There were no differences in accuracy of gaze shift scores and no differences in latency to fixate on the target object. Moreover, gaze shift accuracy scores were not correlated between conditions.

\section{Test Phase}

The primary dependent variable in the test phase was the accuracy score. Thirty-seven infants completed two blocks of test trials, and twelve infants completed one block of trials. To determine whether we could combine the data from infants who completed one and two test blocks, we examined whether infants who completed two test blocks performed differently across blocks (see Table 1 for the accuracy scores during blocks 1 and 2). For infants who completed both blocks, we found no difference in performance between block 1 and 2 (caregiver condition: $t(36)=-0.91, p=0.37, d=0.15$; stranger condition: $t(36)=-0.18, p=0.91, d=0.02)$. Therefore, the accuracy scores were averaged over the two blocks for the infants completing two blocks of trials. For the twelve infants who completed only one block of trials, the values for the single block were used.

\section{Table 1}

Accuracy scores during test phase for each block in each condition

\begin{tabular}{cll} 
Block & Caregiver & Stranger \\
\hline 1 & $0.54(0.03)$ & $0.52(0.04)$ \\
2 & $0.59(0.04)$ & $0.52(0.05)$ \\
\hline
\end{tabular}

Note. Data are reported as mean (standard error). The accuracy score was calculated by dividing the total amount of time spent looking at the target object by the total time spent looking at either 
the target or distractor objects during the analysis window. The table only includes infants that completed both blocks.

A three-way ANCOVA was conducted on accuracy scores, with one within subjects factor: condition (caregiver or stranger), two between-subjects factor: number of blocks completed (one or two) and gender of the caregiver/stranger pair (male or female), and one covariate: mean-centered age. The analysis revealed a significant main effect of condition controlling for infant age, $F(1,44)=6.50, p=0.01, \eta_{p}^{2}=0.13$. Infants' proportion of looking to the target in the caregiver condition $(M=0.56, S E=0.03)$ was significantly greater than their looking in the stranger condition $(M=0.49, S E=0.03)$. There was no significant effect of number of blocks completed $(F<1)$, age $(F<1)$, or gender of the caregiver/stranger pair $(F(1,44)$ $\left.=1.82, p=0.19, \eta_{p}^{2}=0.04\right)$. There were also no significant interactions of number of blocks completed by gender of the caregiver/stranger pair, $F(1,44)=1.05, p=0.31, \eta_{p}^{2}=0.02$, condition by number of blocks, $F<1$, condition by gender of the caregiver/stranger pair, $F(1,44)=2.09, p=$ $0.16, \eta_{p}^{2}=0.05$, or condition by number of blocks by gender of the caregiver/stranger pair, $F<1$.

Next, we compared the accuracy scores against chance (where chance equals 0.5 because of the presence of two objects on the screen). The proportion of looking to the target was significantly different from chance in the caregiver condition, $t(48)=2.51, p=0.02, d=0.36$, but proportion of looking to the target did not significantly differ from chance in the stranger condition, $t(48)=-0.43, p=0.67, d=0.06$. Therefore, infants only showed a preference for the previously labeled object when their caregiver cued the target location.

Because we were particularly interested in how gaze following relates to learning, we examined the gaze data from the training phase and its relation to looking in the test phase in more detail. Based on the gaze shift accuracy scores from the training phase, we used a median 
split to categorize infants as high and low gaze followers for each director separately. Recall that infants' gaze shift scores across director conditions were not correlated. Because of this, infants could not simply be classified as high and low gaze followers overall. The classification was specific to the director.

Caregiver condition. Based on the median split, the low gaze followers had gaze shift scores of 0.50 or less, and high gaze followers had accurate gaze shift scores of 0.54 or greater (see Figure 3). In other words, the best gaze follower in the low gaze following group followed her caregiver's gaze to the target object on $50 \%$ of her gaze shifts, and the worst gaze follower in the high gaze following group followed her caregiver's gaze to the target object on 54\% of her gaze shifts. We performed an independent samples $t$-test to compare the proportion of looking to the target object after the label onset at test for the infants classified as high and low gaze followers based on their performance during the training phase. The proportion of looking to the target at test for high gaze followers $(M=0.62, S E=0.03)$ was significantly greater than the proportion of looking to the target at test for low gaze followers $(M=0.51, S E=0.04 ; t(47)=$ $2.36, p=0.02, d=0.65$ ), indicating that infants who were skilled at following their caregivers' gaze showed stronger looking at the target object at test than infants who were low gaze followers. Showing a significant preference for the target object after the onset of the label at test suggests that infants learned an association between the label and the target object. Low gaze followers' proportion of looking to the target object at test did not differ significantly from chance, $t(23)=0.15, p=0.89, d=0.03$. However, high gaze followers' proportion of looking to the target object at test did differ significantly from chance, $t(24)=3.68, p=0.001, d=0.75$. Therefore, in the caregiver test trials, the significant proportion of looking to the target found when the high and low gaze followers were collapsed appears to be driven by the high gaze 
followers. Infants only appeared to learn from their caregivers if they were also good at following their caregivers' gaze.

Because the distractor object was never looked at or labeled, we cannot conclude that label learning has occurred solely by examining the $300-1800 \mathrm{~ms}$ window after the label onset. Therefore, in order to ensure that the high gaze followers' performance during the window after the onset of the label at test was in response to the label, we also compared their proportion of looking to the target object during the same window prior to the label onset at test (-1800 to $300 \mathrm{~ms}$ ) against chance. If the proportion of looking to the target object at test significantly differs from chance before hearing the label, then this would suggest that the infants' performance during the window after the onset of the label was reflecting a preference for the object to which their caregivers had looked during the training phase, rather than an association between the label and the object. The proportion of looking to the target object prior to the label onset at test did not significantly differ from chance, $t(24)=1.37, p=0.19, d=0.28$ (see Table 2 for the accuracy scores during the pre-label and post-label onset windows). Therefore, we can conclude that the significant proportion of looking to the target at test seen during the window after the onset of the label was tied to hearing the label for that object.

Table 2

Accuracy scores during test phase for each gaze group in each condition

\begin{tabular}{lllll}
\hline & \multicolumn{2}{c}{ Caregiver } & \multicolumn{2}{c}{ Stranger } \\
\hline Window & Low & High & Low & High \\
\hline Pre-Label Onset & $0.47(0.03)$ & $0.55(0.04)$ & $0.45(0.03)$ & $0.51(0.03)$ \\
Post-Label Onset & $0.51(0.04)$ & $0.62(0.03)$ & $0.43(0.04)$ & $0.54(0.04)$ \\
\hline
\end{tabular}

Note. Data are reported as mean (standard error). The accuracy score was calculated by dividing the total amount of time spent looking at the target object by the total time spent looking at either the target or distractor objects during the analysis window. The pre-label onset window was $1800 \mathrm{~ms}-300 \mathrm{~ms}$ before the onset of the label, and the post-label onset window was $300 \mathrm{~ms}-$ $1800 \mathrm{~ms}$ after the onset of the label. 
Stranger condition. Based on the median split, the low gaze followers had accurate gaze shift scores of 0.50 or less, and high gaze followers had accurate gaze shift scores of 0.60 or greater (see Figure 3). We performed an independent samples $t$-test to compare the proportion of looking to the target object at test during the window after the onset of the label for the infants classified as high and low gaze followers based on their performance during the training phase. The proportion of looking to the target at test for high gaze followers $(M=0.54, S E=0.04)$ was significantly greater than the proportion of looking to the target at test for low gaze followers $(M$ $=0.43, S E=0.04 ; t(47)=2.03, p=0.048, d=0.59$ ). Low gaze followers' proportion of looking to the target object at test did not differ significantly from chance, $t(23)=-1.89, p=0.07, d=$ 0.39. High gaze followers' proportion of looking to the target object at test also did not differ significantly from chance, $t(24)=1.04, p=0.31, d=0.19$. Therefore, infants who were better at following a stranger's gaze were more likely to look to the target object at test during the window after the onset of the label, but this increased gaze following did not result in significant learning when the director was unfamiliar to the infant.

\section{Discussion}

The present research investigates how experience with a person affects how infants' use gaze cues to direct attention and learning. Specifically, we addressed two questions in this study. The first question asked: Do infants use familiar and unfamiliar faces differently to guide their attention when learning new words? The second question then asked: Are these infants more likely to learn a label when directed by a familiar agent than when directed by an unfamiliar agent? The results of this study suggest that although visual attention is not more strongly directed by a familiar than by an unfamiliar face, there is an advantage when using a familiar face to guide learning in 12- to 18-month-old infants. As a group, infants learned an association 
between object and label when the caregiver guided their attention, but failed to learn that association when a stranger guided their attention.

We predicted that infants would follow their caregiver's gaze more accurately and more quickly than a stranger's gaze. Contrary to our first hypothesis, no advantage of familiarity was apparent during the training phase; i.e., infants' allocation of visual attention did not differ based on the familiarity of the director during the training phase. During the training trials, infants looked to the face longer than to either the target or distractor object, and looking time to each area did not differ between conditions. There was also no difference in the accuracy of gaze shifts from the caregiver's face to the target object compared to shifts from the stranger's face to the object. Finally, there was no effect of familiarity on latency of gaze shifts from the director to the target object.

We also predicted that infants would be more likely to learn a label when taught by their caregiver than when taught by a stranger. The findings from the test phase are consistent with our second hypothesis. In the caregiver condition, during testing, infants reliably looked to the previously labeled object after hearing the label. Infants did not reliably look to the target object in the stranger condition. In addition, detailed analyses integrating the training and test phases revealed important differences based on gaze following accuracy during training. Accuracy of gaze following was not correlated for the caregiver and stranger trials; therefore, we examined the high and low gaze followers to each director separately. In the caregiver condition, high gaze followers showed a greater proportion of looking to the target object at test than low gaze followers. Infants who more accurately used their caregivers' faces to guide their attention toward the target object during training reliably looked at the target object after hearing its label. Learning was not apparent in the caregiver condition for infants who were less accurate in using 
their caregivers' faces to guide their attention during training. In the stranger condition, high gaze followers tended to show a greater proportion of looking to the target object at test than low gaze followers. However, neither group showed reliable evidence of learning (i.e., a significant proportion of looking to the target object).

In summary, the proportion of looking to the target object at test was related to the accuracy of gaze following during training, such that infants better at gaze following also exhibited a greater proportion of looking to the target object at test, regardless of condition. However, learning was only evident in the high gaze followers in the caregiver condition. No learning was evident in the low gaze followers in the caregiver condition, the low gaze followers in the stranger condition, or the high gaze followers in the stranger condition.

Infants in the current study showed many similarities across conditions in their looking patterns during training: they looked at the stranger's and the caregiver's faces for an equivalent amount of time, looked at the objects for an equivalent amount of time, and shifted attention from the face to the objects as accurately and as quickly in each condition. However, infants only looked reliably at the target object during testing when they were initially cued by their caregiver, a person from whom they have prior experience learning. Our results suggest that being good at following the director's gaze provides infants with an advantage, and having prior experience with the director provides infants with an additional boost. Why might this occur, especially when the only difference is the visual identity of the director? According to the Directed Attention Model, infants can identify a familiar face more quickly than an unfamiliar face, and this rapid identification may allow them to use a familiar face more efficiently than an unfamiliar face (Hoehl et al., 2009; Reid \& Striano, 2007). The Directed Attention Model suggests that in order to gather and use social information an infant must: (1) detect a social 
agent based on biological features (e.g., eyes, motion); (2) identify the social agent based on individual characteristics of the face, for example; (3) detect the focus of the agent's attention in relation to oneself; (4) detect the focus of the agent's attention in relation to objects in the environment; and, (5) prepare a response. It is possible that the familiarity of the caregiver allowed for this second step of the Directed Attention Model to be completed faster when the director was the caregiver than when the director was a stranger. The advantage gained at the second step of this process may have subsequently sped up the remaining three steps. Although we failed to see an advantage in detecting the focus of the agent's attention when the agent was familiar, we did see an advantage in the later stage of preparing and providing a response (learning an association, in this case).

Demonstrating an advantage in learning from a familiar source raises the question of why we did not see an advantage of familiarity in gaze following. Looking time results often fail to find differences that are seen in ERP findings (e.g., de Haan \& Nelson, 1997; Wahl, Michel, Pauen, $\&$ Hoehl, 2012). Therefore, it is possible that our measure was not sensitive enough to reflect the processing differences that were occurring during training. The enhanced processing could have occurred on a covert (rather than overt) level, and thus may not have been possible to capture these differences using eye tracking. Perhaps infants' covert attention was more heightened when their caregiver directed their attention than when a stranger did so. While the Directed Attention Model provides a potential framework for interpreting the results, the current findings could be evidence against the assumption that rapid identification of familiar faces allows infants to more easily follow gaze. It is possible that we failed to see a difference during gaze following because familiarity does not provide an advantage at this intermediate step. Future research using different methodologies, such as ERP, will help explore these possibilities. 
Our findings from the training phase were inconsistent with those of Gredebäck et al. (2010). Infants in our study exhibited similar latencies when shifting from the caregiver's face and when shifting from the stranger's face. In contrast, Gredebäck et al. reported longer latencies for infants in the caregiver condition in comparison to infants in the stranger condition in 8-monthold infants. We also saw no effect of familiarity on accuracy of gaze following, whereas Gredebäck et al. found more gaze following in the 6-month-old infants in the stranger condition than the 6-month-old infants in the mother condition. However, it should be noted that gaze following was evident at 4,6 , and 8 months, but the difference between conditions was only found at 6 months. What could account for the difference in these findings? First, the design used in Gredebäck et al.'s experiment was more naturalistic than the design of the current study. Because of this, the directors were able to use vocal cues to capture infants' attention throughout the experiment. In the current experiment, an audio track pre-recorded by a single actress was used for the entiretly of the experiment. Thus, the only difference between the conditions in the current experiment was the visual familiarity of the faces directing attention. It is, therefore, possible that Gredebäck et al.'s results were driven by the novel attention-grabbing vocal cues of the stranger. Another important difference across experiments is the mode of presentation. We might find a different pattern of results using in-person training rather than video recorded training (as in language learning research by Kuhl, Tsao, \& Liu, 2003). In addition, the infants tested in the current study were older than the infants tested in Gredebäck et al.'s study, so conflicting results could also reflect developmental differences in how infants use familiar and unfamiliar people to guide their attention. Indeed, there is evidence of developmental differences in how infants and young children respond to images of familiar and unfamiliar faces. For example, although 6-month-olds devote more attention (measured by ERP) towards pictures of 
their caregiver compared to a stranger (de Haan \& Nelson, 1997), 3- to 4-year-olds display the opposite pattern (Dawson et al., 2002). The current experiment could be reflecting a similar transition in development.

The results from the test phase of our experiment, are, by contrast, consistent with the results of Hoehl et al.'s (2012) research in which 4-month-old infants showed evidence of more thorough object processing when directed by their caregiver rather than a stranger. Although the infants in Hoehl et al.'s experiment were much younger than the infants tested in the current study, the design was somewhat similar. That is, the infants watched the experiment on a screen without the caregivers' vocal cues. The absence of the director's vocal cues forced the infants to rely on the visual differences in the directors only. In the absence of any additional social cues, an advantage in the caregiver condition was evident.

In the present experiment, a consistent voice was used to label objects in the stranger and the caregiver condition. This afforded careful control over the vocal characteristics of the labeling and allowed us to specifically examine the effects of gaze cueing during learning, separately from the effects of an individual's vocal cues. However, it does raise the possibility that infants responded to the mismatch between the familiar face and unfamiliar voice and this could have enhanced attention and learning. This explanation is unlikely, however, as we found no evidence of differences in looking time during caregiver versus stranger training trials.

The goal of this study was not to investigate whether children could follow gaze on a screen and learn words; nor was it only investigating whether children could learn from their mother and a stranger, as evidence for both already exists in the literature. The current procedure intentionally combined multiple processing challenges for the infants (i.e., two labelers each 
teaching a novel word in the same block of trials) in order to specifically investigate what cue the infants would prioritize when overloaded in the context of this much cognitive demand.

In the current study, accuracy of gaze following was not correlated when infants followed the gaze of their caregivers or of strangers, suggesting that gaze following ability was not a stable characteristic of individual infants, but rather varied across contexts. This was a surprising finding, as it suggests that infants may not simply be "good" or "bad" gaze followers. Future studies will need to examine how individual infants follow the gaze of different cuers under different conditions in more detail to better unpack this.

Another important finding from this study arose from examining performance during training in relation to performance at test. Not only do our results support the idea that a familiar cue enhances learning compared to an unfamiliar cue, they also highlight the importance of an infant's ability to take advantage of a cue. Regardless of the condition, if an infant was better able to use the director to guide her attention to the target object during training, that infant was more likely to look to the target object at test. This provides direct evidence that infants' ability to use a gaze cue during training relates to their ability to display learning of the information that was cued. One consideration to make when interpreting this finding is that we specifically tested 12- to 18-month-old infants so that we could test the relation between gaze following and word learning because previous studies demonstrated a link between gaze following ability and vocabulary (Brooks \& Meltzoff, 2005; Carpenter et al., 1998; Morales et al., 2000). Therefore, the results may not be generalizable to younger infants who are not rapidly learning labels or to domains outside of language.

The findings from this experiment suggest a number of directions for further research. In the present experiment, we used a paradigm that had high experimental control, but was not very 
naturalistic. By adding more naturalistic features (e.g., familiar voices with the familiar faces, gestural cues, contingent interaction), we will gain a better understanding of how infants learn from familiar and unfamiliar people in their natural environments. Adding these additional cues may strengthen the differences in processing between the caregiver and stranger conditions. In addition, future research should include more fathers as stimuli. Although not expected, it is possible that the effects reported here would differ if the caregivers used were primarily male. Another important avenue for future research is to examine how gaze following relates to learning using a larger sample size. The sample size of the gaze following groups $(\mathrm{n}=24$ and $n=25)$ in the current study was significantly reduced from the total sample size $(n=49)$. Although a sample size of 25 per group is common in infant studies, extra caution should nonetheless be used when interpreting any null results from these analyses.

In conclusion, we found that infants used familiar and unfamiliar faces similarly to guide their attention during training, but the same behavior during training resulted in differing performance at test. Learning was only evident in the caregiver condition and only in the infants who were most skilled at following their caregiver's gaze. The results of the current study suggest that both the familiarity of the cuer, and the infant's own ability to follow the gaze of the cuer, play important roles in the infant's learning in this task. This suggests that infants' social experiences interact with their individual abilities to facilitate the way that they learn from people. More broadly, our findings indicate one way that infants' experiences with their caregivers support learning. Input from a familiar person, and the ability to take advantage of that input, help to set the stage for rich learning opportunities starting from early in development. 


\section{References}

Baldwin, D. A., Markman, E. M., Bill, B. B., Desjardins, R. N., Irwin, J. M., \& Tidball, G. (1996). Infants' reliance on a social criterion for establishing word-object relations. Child Development, 67(6), 3135-53. http://doi.org/10.2307/1131771

Bannard, C., \& Tomasello, M. (2012). Can we dissociate contingency learning from social learning in word acquisition by 24-month-olds? PloS One, 7(11), e49881. http://doi.org/10.1371/journal.pone.0049881

Barrera, M. E., \& Maurer, D. (1981). Recognition of Mother's Photographed Face Infant by the Three-Month-Old. Child Development, 52(2), 714-716. http://doi.org/10.2307/1129196

Brooks, R., \& Meltzoff, A. N. (2005). The development of gaze following and its relation to language. Developmental Science, 8(6), 535-43. http://doi.org/10.1111/j.14677687.2005.00445.x

Bushnell, I. W. R. (2001). Mother's face recognition in newborn infants: learning and memory. Infant and Child Development, 10(1-2), 67-74. http://doi.org/10.1002/icd.248

Bushnell, I. W. R., Sai, F., \& Mullin, J. T. (1989). Neonatal recognition of the mother's face. British Journal of Developmental Psychology, 7, 3-15. http://doi.org/10.1111/j.2044835x.1989.tb00784.x

Butterworth, G., \& Jarrett, N. (1991). What minds have in common is space: Spatial mechanisms serving joint visual attention in infancy. British Journal of Developmental Psychology, 9(1), 55-72. http://doi.org/10.1111/j.2044-835X.1991.tb00862.x

Carpenter, M., Nagell, K., Tomasello, M., Butterworth, G., \& Moore, C. (1998). Social cognition, joint attention, and communicative competence from 9 to 15 months of age. Monographs of the Society for Research in Child Development, 63(4). http://doi.org/10.2307/1166214

D'Entremont, B. D. (2000). A perceptual-attentional explanation of gaze following in 3- and 6month-olds. Developmental Science, 3(3), 302-311. http://doi.org/10.1111/14677687.00124

D’Entremont, B. D., Hains, S. M. J., \& Muir, D. W . (1997). A Demonstration of Gaze Following in 3- to 6-Month-Olds. Infant Behavior \& Development, 20(4), 569-572. http://doi.org/10.1016/s0163-6383(97)90048-5

Datavyu Team. (2014). Datavyu: A Video Coding Tool. Databrary Project, New York University. http://datavyu.org.

Dawson, G., Carver, L., Meltzoff, A. N., Panagiotides, H., McPartland, J., \& Webb, S. J. (2002). Neural correlates of face and object recognition in young children with autism spectrum disorder, developmental delay, and typical development. Child Development, 73(3), 700 717. http://doi.org/10.1111/1467-8624.00433

de Bordes, P. F., Cox, R. F. a, Hasselman, F., \& Cillessen, A. H. N. (2013). Toddlers' gaze following through attention modulation: intention is in the eye of the beholder. Journal of Experimental Child Psychology, 116(2), 443-52. http://doi.org/10.1016/j.jecp.2012.09.008

de Haan, M., \& Nelson, C. A. (1997). Recognition of the Mother's Face by Six-Month-Old 
Infants : A Neurobehavioral Study. Child Development, 68(2), 187-210. http://doi.org/10.2307/1131845

Deak, G. O., Flom, R. A., \& Pick, A. D. (2000). Effects of Gesture and Target on 12- and 18Month-Olds' Joint Visual Attention to Objects in Front of or Behind Them. Developmental Psychology, 36(4), 511-523. http://doi.org/10.1037/fflOI

Delaney, H. D., \& Maxwell, S. E. (1981). On Using Analysis of Covariance in Repeated Measures Designs. Multivariate Behavioral Research, 16(1), 105-123. http://doi.org/10.1207/s15327906mbr1601_6

E-Prime 2.0. (2012). Pittsburgh, PA: Psychology Software Tools, Inc.

Farroni, T., Massaccesi, S., \& Johnson, M. H. (2004). Gaze Following in Newborns. Infancy, 5(1), 39-60. http://doi.org/10.1207/s15327078in0501_2

Fernald, A., Perfors, A., \& Marchman, V. A. (2006). Picking up speed in understanding: Speech processing efficiency and vocabulary growth across the 2 nd year. Developmental Psychology, 42(1), 98-116. http://doi.org/10.1037/0012-1649.42.1.98

Fernald, A., Pinto, J. P., Swingley, D., Weinberg, A., \& McRoberts, G. W. (1998). Rapid gains in speed of verbal processing by infants in the 2nd year. Psychological Science, 9(3), 228231. http://doi.org/10.1111/1467-9280.00044

Fernald, A., Zangl, R., Portillo, A. L., \& Marchman, V . A. (2008). Looking while listening: Using eye movements to monitor spoken language comprehension by infants and young children. In I. A. Sekerina, E. M. Fernandez, \& H. Clahsen (Eds.), Developmental Psycholinguistics: On-line methods in children's language processing (pp. 97-135). John Benjamins.

Field, T. M., Cohen, D., Garcia, R., \& Greenberg, R. (1984). Mother-stranger face discrimination by the newborn. Infant Behavior and Development, 7(1), 19-25. http://doi.org/10.1016/S0163-6383(84)80019-3

Goren, C., Sarty, M., \& Wu, P. Y. K. (1975). Visual Following and Pattern Discrimination of Face-like Stimuli by Newborn Infants. Pediatrics, 56, 544-549.

Gredebäck, G., Fikke, L., \& Melinder, A. (2010). The development of joint visual attention: a longitudinal study of gaze following during interactions with mothers and strangers. Developmental Science, 13(6), 839-48. http://doi.org/10.1111/j.1467-7687.2009.00945.x

Haith, M. M., Wentworth, N., \& Canfield, R. L. (1993). The formation of expectations in early infancy. Advances in Infancy Research, 8, 251-297.

Hirotani, M., Stets, M., Striano, T., \& Friederici, A. D. (2009). Joint attention helps infants learn new words: event-related potential evidence. Neuroreport, 20(6), 600-5. http://doi.org/10.1097/WNR.0b013e32832a0a7c

Hoehl, S., Reid, V. M., Parise, E., Handl, A., Palumbo, L., \& Striano, T. (2009). Looking at eye gaze processing and its neural correlates in infancy-implications for social development and autism spectrum disorder. Child Development, 80(4), 968-85. http://doi.org/10.1111/j.14678624.2009.01311.x

Hollich, G. J., Hirsh-pasek, K., Golinkoff, R. M., Rebecca, J., Brown, E., Chung, H. L., ... Bloom, L. (2000). Breaking the Language Barrier: An Emergentist Coalition Model for the 
Origins of Word Learning. Monographs of the Society for Research in Child Development, 65(3), 1-135.

Johnson, M. H., Dziurawiec, S., Ellis, H., \& Morton, J. (1991). Newborns’ preferential tracking of face-like stimuli and its subsequent decline. Cognition, 40(1-2), 1-19. http://doi.org/10.1016/0010-0277(91)90045-6

Kuhl, P. K., Tsao, F.-M., \& Liu, H.-M. (2003). Foreign-language experience in infancy: effects of short-term exposure and social interaction on phonetic learning. Proceedings of the National Academy of Sciences of the United States of America, 100(15), 9096-101. http://doi.org/10.1073/pnas.1532872100

Moore, C., Angelopoulos, M., \& Bennett, P. (1997). The role of movement in the development of joint visual attention. Infant Behavior and Development, 20(1), 83-92. http://doi.org/10.1016/S0163-6383(97)90063-1

Moore, C., Angelopoulos, M., \& Bennett, P. (1999). Word learning in the context of referential and salience cues. Developmental Psychology, 35(1), 60-8. Retrieved from http://www.ncbi.nlm.nih.gov/pubmed/9923464

Morales, M., Mundy, P., Delgado, C. E. F., Yale, M., Messinger, D., Neal, R., \& Schwartz, H. K. (2000). Responding to Joint Attention Across the 6- Through 24-Month Age Period and Early Language Acquisition. Journal of Applied Developmental Psychology, 21(3), 283298. http://doi.org/10.1016/S0193-3973(99)00040-4

Pascalis, O., de Schonen, S., Morton, J., Deruelle, C., \& Fabre-Grenet, M. (1995). Mother's face recognition by neonates: A replication and an extension. Infant Behavior and Development, 18(1), 79-85. http://doi.org/10.1016/0163-6383(95)90009-8

Reid, V. M., \& Striano, T. (2007). The directed attention model of infant social cognition. European Journal of Developmental Psychology, 4(1), 100-110. http://doi.org/10.1080/17405620601005648

Senju, A., \& Csibra, G. (2008). Gaze following in human infants depends on communicative signals. Current Biology : CB, 18(9), 668-71. http://doi.org/10.1016/j.cub.2008.03.059

Von Hofsten, C., Dahlstrom, E., \& Fredriksson, Y. (2005). 12-Month-Old Infants' Perception of Attention Direction in Static Video Images. Infancy, 8(3), 217-231. http://doi.org/10.1207/s15327078in0803_2

Wagner, J. B., Luyster, R. J., Yim, J. Y., Tager-Flusberg, H., \& Nelson, C. a. (2013). The role of early visual attention in social development. International Journal of Behavioral Development, 37(2), 118-124. http://doi.org/10.1177/0165025412468064

Wahl, S., Michel, C., Pauen, S., \& Hoehl, S. (2012). Head and eye movements affect object processing in 4-month-old infants more than an artificial orientation cue. The British Journal of Developmental Psychology, 31(Pt 2), 212-30. http://doi.org/10.1111/bjdp.12001 